\title{
Planar dynamics of large-deformation rods under moving loads
}

\author{
X.W. Zhao ${ }^{\mathrm{a}, \mathrm{b}, 1}$, G.H.M. van der Heijden ${ }^{\mathrm{a}, 1}$ \\ ${ }^{a}$ Department of Civil, Environmental \& Geomatic Engineering, University College London, London WC1E 6BT, UK \\ ${ }^{b}$ School of Aerospace Engineering and Applied Mechanics, Tongji University, Shanghai, People's Republic of China
}

\begin{abstract}
We formulate the problem of a slender structure (a rod) undergoing large deformation under the action of a moving mass or load motivated by inspection robots crawling along bridge cables or high-voltage power lines. The rod is described by means of geometrically exact Cosserat theory which allows for arbitrary planar flexural, extensional and shear deformations. The equations of motion are discretised using the generalised- $\alpha$ method. The formulation is shown to handle the discontinuities of the problem well. Application of the method to a cable and an arch problem reveals interesting nonlinear phenomena. For the cable problem we find that large deformations have a resonance detuning effect on cable dynamics. The problem also offers a compelling illustration of the Timoshenko paradox. For the arch problem we find a stabilising (delay) effect on the in-plane collapse of the arch, with failure suppressed entirely at sufficiently high speed.

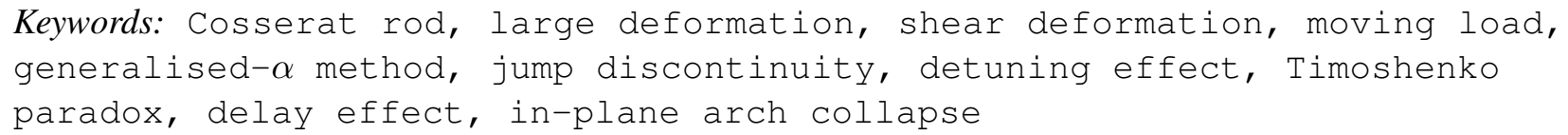

\section{Introduction}

The problem of a continuously distributed system carrying a moving concentrated mass has broad applications in mechanics and engineering, including space tethers, satellite antennas, launch systems, robotic arms [1], cranes [2], flexible manipulators [3], high-speed train railroads and highway bridges with moving vehicles $5 \quad[4,5]$.

The classical example of a moving-mass problem is the idealisation of a vehicle-bridge system. In this case the moving vehicle is usually treated as a moving force, or load, of constant magnitude, while the bridge is modelled, for instance, as a simply-supported beam. This problem is therefore more accurately described as a moving-load problem. The moving load assumption does not take into account the inertial forces of the moving mass and the effect of the beam on the mass. For an overview of the sizeable early literature on the vibration theory of moving-load problems we refer to Fryba's monograph [6]. More recent works on moving loads or masses travelling along beams are [7-9], while more specific studies include moving loads or masses along curved beams or arches [10-12], inclined beams [13], multi-span beams [14, 15] and tapered beams [16]. Meanwhile, loads or masses travelling along cables (modelled as strings, i.e., without bending stiffness), are studied in [17-19]. See also the review in [20].

All the above works restrict themselves to beams or cables undergoing small deflections (in [8] moderately large deflections are considered) and all consider only two-dimensional bending vibrations (in [19] equations are first developed for three-dimensional deformations but these are then condensed to a planar model). With the current drive to use thinner and lighter materials, in order to save material and reduce costs, large deformations become increasingly important.

\footnotetext{
${ }^{1}$ Current address: School of Aerospace Engineering, Tsinghua University, Beijing, People's Republic of China

${ }^{2}$ Corresponding author. E-mail: g.heijden@ucl.ac.uk
} 
In this paper we formulate the moving-load problem for a geometrically-exact Cosserat rod that can undergo arbitrary two-dimensional flexural, extensional and shear deformations. This planar case seems to cover most moving-load applications. The restriction to the moving-load problem is justified by the fact that in cases with large deflections the speeds are likely to be relatively small so that inertial effects can be neglected. As a typical application we are here thinking of inspection robots crawling along bridge cables or high-voltage power lines [21]. We leave the proper treatment of the moving mass problem, with inertia included, for a future publication.

Usually in the literature when (moderately) large deformations are considered, approximate equations are derived (usually involving a geometrically nonlinear strain-displacement relation [8]). These approximate equations are often arguably more complicated (and less transparent) than the geometrically exact equations. Moreover, these equations then still need to be solved numerically, typically by employing a Galerkin expansion (using on the order of 10 terms) [8, 19]. These Galerkin expansions are well known to suffer from lack of uniform convergence (Gibbs phenomenon) in problems with jump discontinuities, as occur in the internal force in moving-load problems [22, 23].

Purely numerical methods using time-stepping algorithms directly on the equations of motion without initial approximation is often avoided because of convergence limitations [24]. However, sophisticated current numerical methods can now solve the exact nonlinear equations with little difficulty and this is the approach taken in this paper.

Cosserat theory describes the evolution of a material director frame, attached to the cross-section of the rod, as it moves along the rod and in time. By introducing an angle to parametrise the director frame we obtain a formulation free from kinematic constraints (and from Euler-angle singularities). In this reduced description it is natural to include the centreline integration within the full discretisation. This gives a more efficient and accurate scheme than existing three-dimensional formulations that use post-processing (i.e., updating of the rotation matrix, typically by using the Rodrigues formula, to compute the directors, and integrating the tangent vector, typically by using the trapezoidal rule) to obtain rod shapes [25, 26].

45 As is natural with large deformations, we are not only interested in natural modes of vibration but in transient dynamics of large amplitude. We therefore discretise the equations of motion using the generalised- $\alpha$ method in both the spatial and temporal domain. This numerical method is found to have good convergence and stability properties for moving-load problems. Of course our approach can also be applied to cables and rods with fixed attached masses. Point loads or masses greatly complicate the description of cable motion because of singularity problems in internal forces [18, 22-24].

The paper is organised as follows. In Section 2 we introduce the planar formulation of Cosserat rod dynamics, while the numerical discretisation is presented in Section 3. In Section 4 we then apply the theory to a few planer problems. The first of these is a 'pendulum test' in which we drop a hinged rod under gravity from a horizontal position for a sequence of bending stiffnesses approaching the rigid pendulum limit. This problem is also used to verify energy conservation of the numerical scheme to second-order accuracy. We then apply our method to both a cable and an arch problem. We find interesting new nonlinear behaviour induced by moving loads on flexible structures. For the cable problem we find that large deformations have a resonance detuning effect. For the arch problem we find the moving load to have a stabilising effect. Some similar stabilisation effect of the speed of the load (a decrease of the midspan displacement of a cable under an increase of the speed of a moving mass) is noted with surprise in [19]. This behaviour is also observed in [16]. Both these results are for small deflections. In our present case, with large deflections, the effect is much stronger and we find that an arch that would collapse under a given quasi-static load will stand if it is traversed by the same load moving at sufficiently high speed. Conclusions follow in Section 5.

\section{Formulation of Cosserat rod dynamics}

In Cosserat theory the configuration of a rod deforming in the plane (by arbitrary bending, shear and extension, but not torsion) is determined by two vector-valued functions $\left(\mathbf{r}(s, t), \mathbf{d}_{1}(s, t)\right) \in \mathbb{R}^{2} \times \mathbb{R}^{2}$ of arclength $s \in[0, L]$ of the unstressed rod and time $t$ ( $L$ being the length of the unstressed rod). Here $\mathbf{r}$ represents the centreline of the rod, while $\mathbf{d}_{1}$ is a unit vector in the plane of the deformed rod pointing along a material line in 


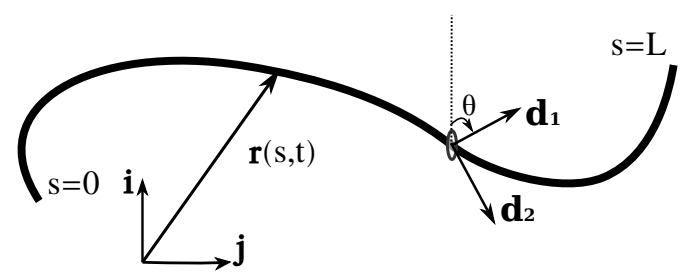

Figure 1: Coordinate systems for a Cosserat rod. $\mathbf{d}_{1}$ and $\mathbf{d}_{2}$ are orthogonal and in the plane of the deformed rod, with $\mathbf{d}_{1}$ pointing along a material line in the rod's cross-section. The angle $\theta$ is measured from the local vertical $\mathbf{i}$.

the rod's cross-section. We also introduce the unit normal vector to the cross-section at $s, \mathbf{d}_{2}$, and parametrise the director (or body) frame $\left\{\mathbf{d}_{1}, \mathbf{d}_{2}\right\}$ by means of the angle $\theta$ as follows

$$
\begin{aligned}
& \mathbf{d}_{1}=\cos \theta \mathbf{i}+\sin \theta \mathbf{j}, \\
& \mathbf{d}_{2}=-\sin \theta \mathbf{i}+\cos \theta \mathbf{j},
\end{aligned}
$$

relative to the fixed inertial frame $\{\mathbf{i}, \mathbf{j}\}$ (see Fig. 1). Note that since the rod is shearable the director $\mathbf{d}_{2}$ will in general not be equal to the tangent vector, $\mathbf{r}^{\prime}(s, t):=\partial \mathrm{r}(\mathrm{s}, \mathrm{t}) / \partial \mathrm{s}=: \partial_{\mathrm{s}} \mathrm{r}(\mathrm{s}, \mathrm{t})$, to the rod. In fact, for this tangent vector we will write

$$
\mathbf{r}^{\prime}=\mathbf{v}
$$

with components $v_{1}$ and $v_{2}$ in the body frame: $\mathbf{v}=v_{1} \mathbf{d}_{1}+v_{2} \mathbf{d}_{2}$. (We shall generally use subscripts ' 1 ' and ' 2 ' to indicate components of any vector in the body frame and subscripts ' $x$ ' and ' $y$ ' to indicate components in the fixed frame $\{\mathbf{i}, \mathbf{j}\}$.) $v_{1}$ and $v_{2}-1$ are the shear and extensional strains in the rod, respectively.

The equations of motion of the rod are

$$
\begin{aligned}
& \mathbf{F}^{\prime}+\mathbf{f}=\rho A \ddot{\mathbf{r}}, \\
& M^{\prime} \mathbf{k}+\mathbf{r}^{\prime} \times \mathbf{F}+m \mathbf{k}=\rho I \ddot{\theta} \mathbf{k},
\end{aligned}
$$

where $\mathbf{F}$ is the resultant force and $M$ the resultant bending moment acting at the cross-section at $s, \mathbf{f}$ is any external force, $m$ any external moment, $\mathbf{k}=\mathbf{i} \times \mathbf{j}$ and an overdot is used for time derivatives. $A$ is the area of the cross-section, $I$ is the second moment of area of the cross-section and $\rho$ is the density of the material.

We finally need to specify the constitutive relations between the forces and the strains and the moment and the curvature $\kappa=\mathbf{d}_{1}^{\prime} \cdot \mathbf{d}_{2}=\theta^{\prime}$ in the rod. We choose the following relations of linear elasticity:

$$
\begin{aligned}
& F_{1}=A_{1} v_{1}, \\
& F_{2}=A_{2}\left(v_{2}-1\right), \\
& M=B\left(\theta^{\prime}-\kappa_{0}\right),
\end{aligned}
$$

where $A_{1}$ is the shear stiffness, $A_{2}$ the axial stiffness and $B$ the bending stiffness, and we allow for a (constant) intrinsic curvature $\kappa_{0}$. For an isotropic and homogeneous material the stiffnesses can be expressed in terms of Young's modulus $E$ and Poisson's ratio $\nu$ as follows: $A_{1}=k A G, A_{2}=A E, B=E I$, where $G=$ $E /[2(1+\nu)]$ is the shear modulus and $k$ is the shear correction factor for which we shall take the common Roark factor $k=9 / 10$ for a circular cross-section. (It may be worth noting that the above theory does not make any assumption about the shape of the rod's cross-section. It may or may not be circular. If it is not then the rod may have a bending stiffness, for bending about $\mathbf{d}_{1}$, different from $B$, but since in this planar theory bending is only about $\mathbf{k}$, this bending stiffness will not enter the equations.)

We write the equations of motion in first-order form by introducing the angular and linear velocities as extra variables:

$$
\begin{aligned}
& \omega=\dot{\mathbf{d}}_{1} \cdot \mathbf{d}_{2}=\dot{\theta}, \\
& \mathbf{u}=\dot{\mathbf{r}} .
\end{aligned}
$$


On differentiating both sides of these equations with respect to $s$ and using Eqs. (3) and (8), we can replace Eqs (9) and (10) by their differentiated form (compatibility equations)

$$
\begin{aligned}
& \frac{\dot{M}}{E I}=\omega^{\prime}, \\
& \dot{\mathbf{v}}=\mathbf{u}^{\prime} .
\end{aligned}
$$

By writing $\mathbf{r}=x \mathbf{i}+y \mathbf{j}$, and using Eqs. (6) and (7) to express the strains $v_{i}$ in terms of the forces $F_{i}$, Eqs (4), (5), (8), (3), (11) and (12) can be written as the following system of nine first-order equations:

$$
\begin{aligned}
\rho A \dot{u}_{1} & =F_{1}^{\prime}-\left(\frac{M}{E I}+\kappa_{0}\right) F_{2}+f_{1}+\rho A \omega u_{2}, \\
\rho A \dot{u}_{2} & =F_{2}^{\prime}+\left(\frac{M}{E I}+\kappa_{0}\right) F_{1}+f_{2}-\rho A \omega u_{1}, \\
\rho I \dot{\omega} & =M^{\prime}+\frac{F_{1} F_{2}}{A_{1}}-\left(1+\frac{F_{2}}{A_{2}}\right) F_{1}+m, \\
0 & =\theta^{\prime}-\frac{M}{E I}-\kappa_{0}, \\
0 & =x^{\prime}-\frac{F_{1}}{A_{1}} \cos \theta+\left(1+\frac{F_{2}}{A_{2}}\right) \sin \theta, \\
0 & =y^{\prime}-\frac{F_{1}}{A_{1}} \sin \theta-\left(1+\frac{F_{2}}{A_{2}}\right) \cos \theta, \\
\frac{\dot{M}}{E I} & =\omega^{\prime}, \\
\frac{\dot{F}_{1}}{A_{1}} & =u_{1}^{\prime}-\left(\frac{M}{E I}+\kappa_{0}\right) u_{2}+\omega\left(1+\frac{F_{2}}{A_{2}}\right), \\
\frac{\dot{F}_{2}}{A_{2}} & =u_{2}^{\prime}+\left(\frac{M}{E I}+\kappa_{0}\right) u_{1}-\omega \frac{F_{1}}{A_{1}} .
\end{aligned}
$$

We can write this system in the compact standard form

$$
\mathbf{M} \dot{\mathbf{y}}+\mathbf{K} \mathbf{y}^{\prime}+\Lambda=\mathbf{0}
$$

in terms of the unknowns $\mathbf{y}=\left(F_{1}, F_{2}, M, \theta, x, y, \omega, u_{1}, u_{2}\right)$ with $\mathbf{M}$ and $\mathbf{K}$ mass and stiffness matrices (constant in the case of a uniform rod). All the nonlinear terms of the system of equations Eqs. (13)-(21) are contained in the load vector $\boldsymbol{\Lambda}$. Note that Eqs (16)-(18) do not contain a time derivative. The mass matrix $\mathbf{M}$ is thus singular. This singularity is not a problem for our numerical solution method, to be discussed in the next section. (The stiffness matrix $\mathbf{K}$ is rendered regular by using the differentiated form of Eqs. (11) and (12) rather than Eqs. (9) and (10) directly.)

In components, Eqs (9) and (10) read

$$
\begin{aligned}
& \dot{\theta}=\omega, \\
& \dot{x}=u_{1} \cos \theta-u_{2} \sin \theta, \\
& \dot{y}=u_{1} \sin \theta+u_{2} \cos \theta,
\end{aligned}
$$

or, in compact standard form,

$$
\mathbf{M}_{c} \dot{\mathbf{y}}+\boldsymbol{\Lambda}_{c}=\mathbf{0} .
$$

These equations act as constraints that fix the spatial integration constants lost by including the differentiated forms Eqs. (11), (12) in our system of equations. They can be applied at an arbitrary spatial point $s$. With 
these three constraints applied, Eqs. (13)-(21) require six boundary conditions for a well-posed boundaryvalue problem. We are not aware of this formulation for geometrically exact deformations of extensible and shearable planar rods appearing anywhere explicitly in the literature.

The equations for an inextensible and unshearable rod are obtained by dropping all terms involving $A_{1}$ or $A_{2}$ (i.e., taking the limit $A_{1} \rightarrow \infty, A_{2} \rightarrow \infty$ ).

\section{Numerical discretisation - the generalized- $\alpha$ method}

The generalised- $\alpha$ method is a Newmark-like implicit time-integration scheme with desirable features such as second-order accuracy, unconditional (linear) stability and controllable numerical dissipation, first proposed by Chung and Hulbert in 1993 to solve linear structural dynamics problems [27]. Erlicher et al. [28] verified the accuracy and stability of the method when applied to nonlinear dynamics problems. We apply the generalised- $\alpha$ method for both spatial and temporal discretisation, as in $[25,26]$.

In the first step we derive the semi-discrete form of Eq. (22) with respect to time as

$$
\left(\mathbf{M} \partial_{t} \mathbf{y}\right)^{1-\alpha_{t}}+\left(\mathbf{K} \partial_{s} \mathbf{y}\right)^{1-\beta_{t}}+\mathbf{\Lambda}^{1-\beta_{t}}(\mathbf{y})=\mathbf{0},
$$

where $(\cdot)^{1-\iota}=(1-\iota)(\cdot)^{i}+\iota(\cdot)^{i-1}$. The parameters $\alpha_{t}$ and $\beta_{t}$ average the inertial term and terms of the stiffness and the load vector of the rod system, respectively, in time. $\mathbf{M}$ and $\mathbf{K}$ are constant in our case. As to the interpretation of the nonlinear $\Lambda^{i}$ term, we choose the generalised trapezoidal rule (one of the three quadrature rules summarised in [28]), which corresponds to setting $\boldsymbol{\Lambda}^{1-\beta_{t}}(\mathbf{y})=\left(1-\beta_{t}\right) \boldsymbol{\Lambda}\left(\mathbf{y}^{i}\right)+\beta_{t} \boldsymbol{\Lambda}\left(\mathbf{y}^{i-1}\right)$. Eq. (27) is then expressed as

$$
\begin{aligned}
\mathbf{M}\left[\left(1-\alpha_{t}\right) \partial_{t} \mathbf{y}^{i}\right. & \left.+\alpha_{t} \partial_{t} \mathbf{y}^{i-1}\right] \\
& +\mathbf{K}\left[\left(1-\beta_{t}\right) \partial_{s} \mathbf{y}^{i}+\beta_{t} \partial_{s} \mathbf{y}^{i-1}\right] \\
& \quad+\left(1-\beta_{t}\right) \boldsymbol{\Lambda}\left(\mathbf{y}^{i}\right)+\beta_{t} \boldsymbol{\Lambda}\left(\mathbf{y}^{i-1}\right)=\mathbf{0} .
\end{aligned}
$$

Similarly, applying the generalised- $\alpha$ method in space, with averaging parameters $\alpha_{s}$ and $\beta_{s}$, gives

$$
\begin{aligned}
\mathbf{M}\{ & \left.\left(1-\alpha_{t}\right)\left[\left(1-\alpha_{s}\right) \partial_{t} \mathbf{y}_{j}^{i}+\alpha_{s} \partial_{t} \mathbf{y}_{j-1}^{i}\right]+\alpha_{t}\left[\left(1-\alpha_{s}\right) \partial_{t} \mathbf{y}_{j}^{i-1}+\alpha_{s} \partial_{t} \mathbf{y}_{j-1}^{i-1}\right]\right\} \\
& +\mathbf{K}\left\{\left(1-\beta_{t}\right)\left[\left(1-\beta_{s}\right) \partial_{s} \mathbf{y}_{j}^{i}+\beta_{s} \partial_{s} \mathbf{y}_{j-1}^{i}\right]+\beta_{t}\left[\left(1-\beta_{s}\right) \partial_{s} \mathbf{y}_{j}^{i-1}+\beta_{s} \partial_{s} \mathbf{y}_{j-1}^{i-1}\right]\right\} . \\
& +\left\{\left(1-\beta_{t}\right)\left[\left(1-\beta_{s}\right) \boldsymbol{\Lambda}_{j}^{i}+\beta_{s} \boldsymbol{\Lambda}_{j-1}^{i}\right]+\beta_{t}\left[\left(1-\beta_{s}\right) \boldsymbol{\Lambda}_{j}^{i-1}+\beta_{s} \boldsymbol{\Lambda}_{j-1}^{i-1}\right]\right\}=\mathbf{0} .
\end{aligned}
$$

In this equation the superscript indicates the time step while the subscript indicates the spatial step, i.e., the node of the discretised curve.

In the second step the $s$ and $t$ derivatives are approximated as

$$
\partial_{t} \mathbf{y}^{i}=\frac{\mathbf{y}^{i}-\mathbf{y}^{i-1}}{\gamma_{t} \Delta t}-\frac{1-\gamma_{t}}{\gamma_{t}} \partial_{t} \mathbf{y}^{i-1}, \quad \partial_{s} \mathbf{y}_{j}=\frac{\mathbf{y}_{j}-\mathbf{y}_{j-1}}{\gamma_{s} \Delta s}-\frac{1-\gamma_{s}}{\gamma_{s}} \partial_{s} \mathbf{y}_{j-1},
$$

where $\gamma_{t}$ and $\gamma_{s}$ are two more averaging parameters and $\Delta s$ and $\Delta t$ are the spatial step size and time step size, respectively. By substituting Eq. (30) into Eq. (29) we obtain a system of algebraic equations in the form

$$
\mathcal{F}_{j}^{i}:=\mathbf{A}\left(\mathbf{y}_{j}^{i}\right)+\mathbf{B}\left(\mathbf{y}_{j-1}^{i}\right)-\zeta \partial_{s}(\mathbf{K y})_{j-1}^{i}-\mathbf{R}_{j-1}^{i-1}=\mathbf{0}
$$

with

$$
\zeta=\frac{\left(1-\beta_{t}\right)\left(1-\beta_{s}-\gamma_{s}\right)}{\gamma_{s}}
$$

Here $\mathbf{A}\left(\mathbf{y}_{j}^{i}\right)$ and $\mathbf{B}\left(\mathbf{y}_{j-1}^{i}\right)$ are nonlinear functions of the unknown variables, while $\mathbf{R}_{j-1}^{i-1}:=\mathbf{R}_{j-1}^{i-1}\left(\mathbf{y}_{j}^{i-1}, \mathbf{y}_{j-1}^{i-1}\right.$, $\left.\partial_{t} \mathbf{y}_{j}^{i-1}, \partial_{t} \mathbf{y}_{j-1}^{i-1}, \partial_{s} \mathbf{y}_{j-1}^{i-1}\right)$ is known once variables at the earlier time step $\mathbf{y}^{i-1}$ are solved. Each set of three parameters $\alpha_{s}, \beta_{s}, \gamma_{s}$ and $\alpha_{t}, \beta_{t}, \gamma_{t}$ can be expressed as functions of $\lambda_{(\cdot)}^{\infty}$,

$$
\alpha_{(\cdot)}=\frac{3 \lambda_{(\cdot)}^{\infty}+1}{2 \lambda_{(\cdot)}^{\infty}-2}, \quad \beta_{(\cdot)}=\frac{\lambda_{(\cdot)}^{\infty}}{\lambda_{(\cdot)}^{\infty}-1}, \quad \gamma_{(\cdot)}=\frac{1}{1-\lambda_{(\cdot)}^{\infty},},
$$


where $\lambda_{(\cdot)}^{\infty} \in[-1,0]$ is the spectral radius of the algorithm defined by $\lambda_{(\cdot)}^{\infty}=\max \left(\left|\lambda_{1}\right|,\left|\lambda_{2}\right|,\left|\lambda_{3}\right|\right)$, the maximum eigenvalue of the amplification matrix in the high-frequency limit $(\omega \Delta t \rightarrow \infty)$. The parametrisation (32) automatically satisfies $\alpha_{(\cdot)} \leq \frac{1}{2}, \beta_{(\cdot)} \leq \frac{1}{2}, \gamma_{(\cdot)} \geq \frac{1}{2}$ and $\alpha_{(\cdot)}-\beta_{(\cdot)}+\gamma_{(\cdot)}=\frac{1}{2}$, the conditions for unconditional stability and second-order accuracy. The remaining parameter, $\lambda_{(\cdot)}^{\infty}$, can then be tuned for any desired numerical dissipation. More details can be found in $[27,29,30]$. Note that $\beta_{(\cdot)}+\gamma_{(\cdot)}=1$ and hence

$$
\zeta=0 .
$$

This allows us to eliminate the terms with $\zeta$ in Eq. (31) thereby significantly simplifying the Jacobian matrix $\partial \mathcal{F}_{j}^{i} / \partial \mathbf{y}_{l}^{k}$ of the nonlinear system of equations without loss of either accuracy or numerical stability. We also commment that unlike the chain problem in [29, 30], we do not suffer any loss of robustness/stability by choosing $\lambda_{s}^{\infty}=\lambda_{t}^{\infty}=-1$, corresponding to the box method ( $1 / 2$ for all six parameters). This gives a stable second-order scheme without numerical damping and will be our method of choice in the following applications.

Also applying the generalised- $\alpha$ method to the constraint Eqs. (26) for temporal discretisation, we get the algebraic equations, at an arbitrary spatial node $k$, in the form

$$
\begin{aligned}
\mathcal{G}_{k}^{i+1}:=\frac{\left(1-\alpha_{t}\right)}{\gamma_{t} \Delta t} \mathbf{M}_{c} \mathbf{y}_{k}^{i+1} & +\left(1-\beta_{t}\right) \boldsymbol{\Lambda}_{c}\left(\mathbf{y}_{k}^{i+1}\right) \\
& -\frac{\left(1-\alpha_{t}\right)}{\gamma_{t} \Delta t} \mathbf{M}_{c} \mathbf{y}_{k}^{i}+\frac{\alpha_{t}+\gamma_{t}-1}{\gamma_{t}} \mathbf{M}_{c} \partial_{t} \mathbf{y}_{k}^{i}+\beta_{t} \boldsymbol{\Lambda}_{c}\left(\mathbf{y}_{k}^{i}\right)=\mathbf{0}
\end{aligned}
$$

where $k \in\left\{1,2, \cdots, N_{s}\right\}, N_{s}$ being the number of spatial nodes. We take $k=1$ and impose the constraint at $s=0$.

We thus have the full set of algebraic equations

$$
\mathcal{H}^{i+1}(\mathbf{y})=\left\{\mathcal{F}_{1}^{i+1}, \mathcal{F}_{2}^{i+1}, \cdots, \mathcal{F}_{N_{s}-1}^{i+1}, \mathcal{G}_{1}^{i+1}\right\}=\mathbf{0}
$$

where $\mathcal{H}^{i+1} \in \mathbb{R}^{6\left(N_{s}-1\right)+3}$. This final system of algebraic equations (34) is solved with the global Newton code NLEQ1 [31]. The detailed Jacobian is given in the Appendix.

\section{Applications}

We apply our formulation to a few planar structures with travelling loads. The applied loads that we consider are gravity and the vertical moving point load of magnitude $N$, so we have $m=0$ and

$$
\mathbf{f}=-(\rho A g+N \delta(s-\sigma)) \mathbf{i}
$$

where $g$ is the gravitational acceleration, $\delta$ is the Dirac- $\delta$ function and $\sigma=\sigma(t)$ is the instantaneous arclength position of the moving point load. Projection onto the director frame gives

$$
\begin{aligned}
& f_{1}=\mathbf{f} \cdot \mathbf{d}_{1}=-(\rho A g+N \delta(s-\sigma)) \cos \theta \\
& f_{2}=\mathbf{f} \cdot \mathbf{d}_{2}=(\rho A g+N \delta(s-\sigma)) \sin \theta
\end{aligned}
$$

for the force components $f_{1}$ and $f_{2}$ in the force balance equations (13) and (14). The load $N$ may be an arbitrary function of time and arclength coordinate $s$, but we shall only consider the case of constant and uniform $N$.

The treatment of the $\delta$-function needs some care, as the position of the point load at a given time $t, \sigma(t)$, will in general not coincide with a spatial node. One approach would be to introduce an extra 'moving node' where the load is located [32], but this would add considerable complexity to the numerical implementation. Another way to deal with this problem is to replace the point load by statically equivalent forces at the nearest grid points on either side of $\sigma$, as proposed in [33]. Instead, we solve this problem by always taking $\Delta t=\Delta s / a$, where $a=\mathrm{d} \sigma / \mathrm{d} t$ is the (constant) speed of the moving load, thereby ensuring that the solution is sampled at times 
when the load is applied exactly at a node. This avoids any (error-introducing) interpolation, is very convenient

to implement and is found to work well. We merely have to replace $N \delta(s-\sigma)$ by the discrete version $N / \Delta s$ at the appropriate node.

In all the following examples the rod is taken to be uniform with circular cross-section and of length $L=1 \mathrm{~m}$ and radius $r=0.005 \mathrm{~m}$, while $\nu=0.25, \rho=1500 \mathrm{~kg} / \mathrm{m}^{3}$ and $g=9.8 \mathrm{~m} / \mathrm{s}^{2}$. We vary Young's modulus $E$ for the aim of tuning flexibility of the structures.

\subsection{The pendulum test - an extensible and shearable rod falling under gravity}

An extensible and shearable rod is pinned at one end and initially placed in a horizontal position and then allowed to fall naturally under gravity (for instance, by pulling away a table or other support from underneath it). The boundary conditions for this problem are given by

$$
\begin{aligned}
s=0: & M=0, & y=0, & z=0, \\
s=L: & F_{2}=0, & F_{3}=0, & M=0 .
\end{aligned}
$$

We show representative snapshots of falling rods with Young's modulus $E=0.25 \mathrm{GPa}$ and $E=210 \mathrm{GPa}$ within the first half falling period in Fig. 2 and Fig. 3, respectively. Fig. 4 shows instantaneous shapes of rods at time $t=0.05 \mathrm{~s}$ with increasing Young's modulus from a low value of $E=0.05 \mathrm{GPa}$ to a high value $E=210 \mathrm{GPa}$, approaching the limit of a rigid pendulum. In all the runs for this example we choose $\Delta t=0.01 \mathrm{~s}$.

The potential energy due to bending, shear, extension and gravity of the rod is given by

$$
V=\int_{0}^{L} \frac{1}{2}\left(\frac{M^{2}}{E I}+\frac{F_{1}^{2}}{A_{1}}+\frac{F_{2}^{2}}{A_{2}}\right) \mathrm{d} s+\int_{0}^{L} \rho A g y \mathrm{~d} s
$$

and kinetic energy by

$$
T=\int_{0}^{L} \frac{1}{2}\left(\rho A u_{1}^{2}+\rho A u_{2}^{2}+\rho I \omega^{2}\right) \mathrm{d} s .
$$

Figs. 5 and 6 demonstrate conservation of the total energy $U=V+T$ of the falling pendulum-like rod ( $E=210 \mathrm{GPa}$ ). The maximum absolute value of $U$ is $3 \times 10^{-5}$, which is consistent with $\mathcal{O}\left(\Delta t^{2}\right)$, as expected for a second-order integration method.

A convergence test is given in Fig. 7 by tracing the end position in the vertical direction $y(L)$ when we take different spatial step sizes. It is seen that step sizes $\Delta s=0.01 \mathrm{~m}$ and $\Delta s=0.1 \mathrm{~m}$ do not lead to any noticeable difference. Thus we deem the numerical method to have converged and we take $\Delta s=0.01 \mathrm{~m}$ in the other runs for this example.

Fig. 8 gives the total length of the stretched falling rod. Rods with higher $E$ values stretch less, as expected. The maximum magnitudes of stretch (defined as the relative extension of the rod) within the first 8 seconds of the drop are given in Table 1 . We present the $\mathbf{d}_{2}$ component of the end velocity, $u_{2}(L)$, in Fig. 9 , which shows the resultant effects of stretch, shear and bending of the full rod length. It oscillates with higher frequency and smaller amplitude when the rod is stiffer.

\subsection{A hanging cable under a moving load}

We consider an inextensible and unshearable cable hanging under gravity (taking $E=0.25 \mathrm{GPa}$ ). Its end tangents are horizontal and prevented from rotation but the right end is free to move along the end-to-end axis (see true view in Fig. 10). The boundary conditions are given by

$$
\begin{array}{llll}
s=0: & \theta=0, & y=0, & z=0, \\
s=L: & F_{2}=0, & \theta=0, & y=0 .
\end{array}
$$

The statically hanging shape of the cable can be computed by solving our equations with mass matrices $\mathbf{M}$ and $\mathbf{M}_{c}$ set to zero in Eqs. (22) and (26). We prepare the cable under external loads consisting of gravity and 


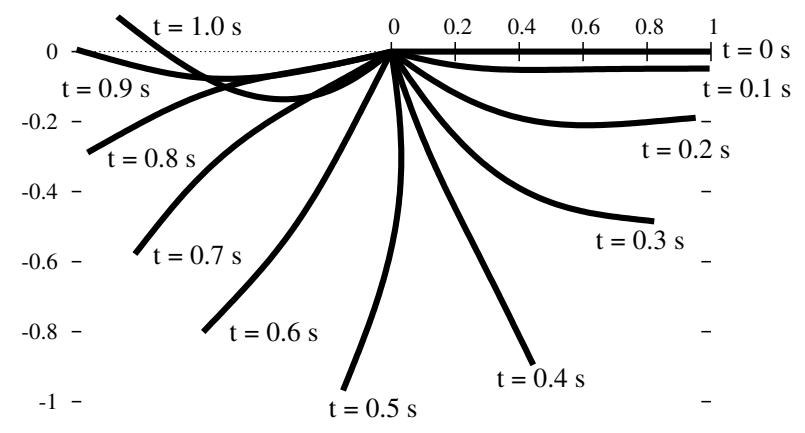

Figure 2: Snapshots of a falling rod with Young's modulus $E=$ $0.25 \mathrm{GPa}$

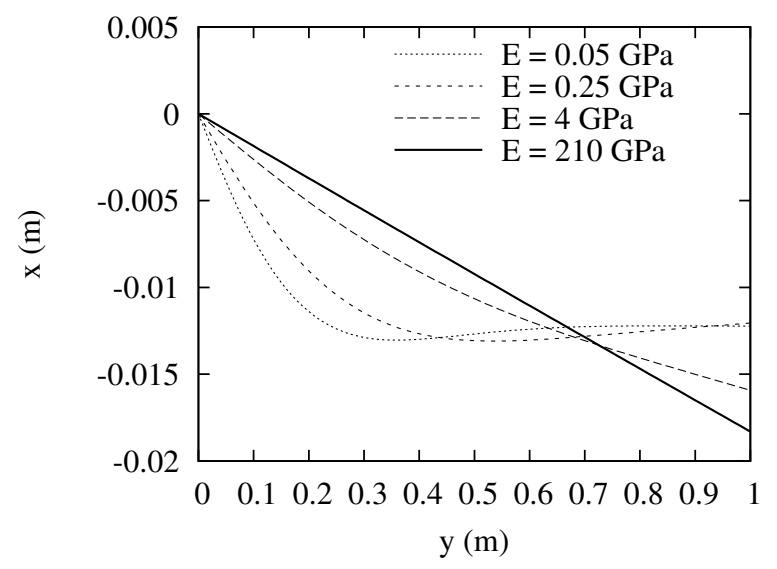

Figure 4: Shapes of falling rods at $t=0.05 \mathrm{~s}$.

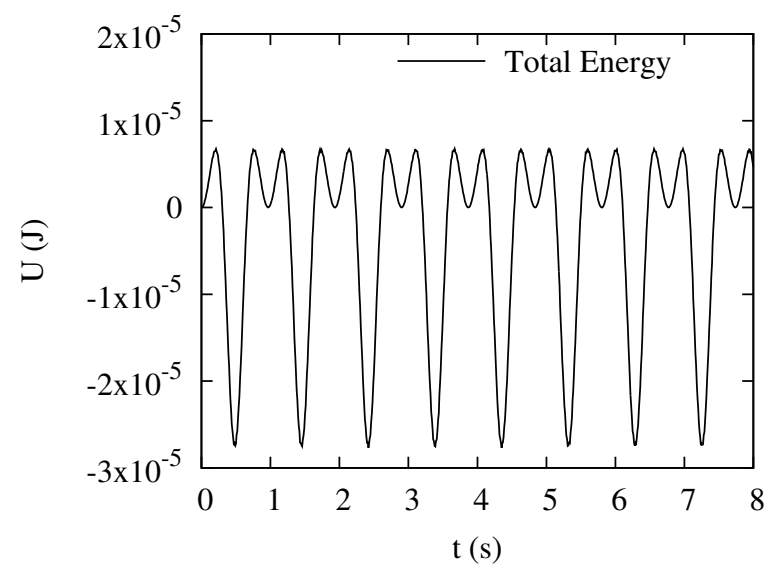

Figure 6: Total energy of a falling rod with Young's modulus $E=210 \mathrm{GPa}$.

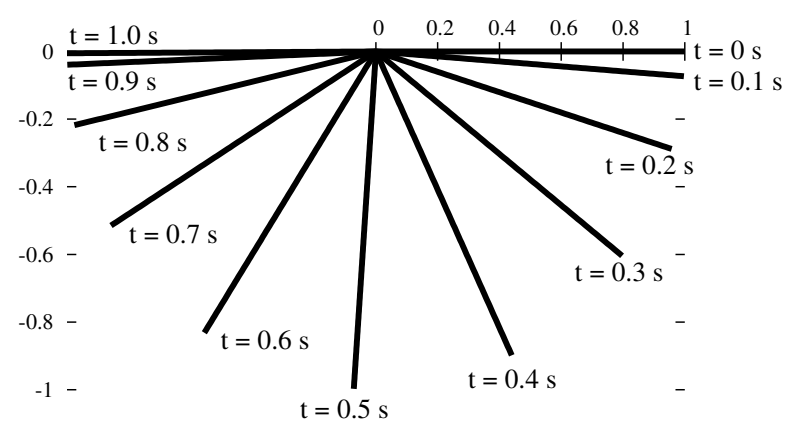

Figure 3: Snapshots of a falling rod with Young's modulus $E=$ $210 \mathrm{GPa}$

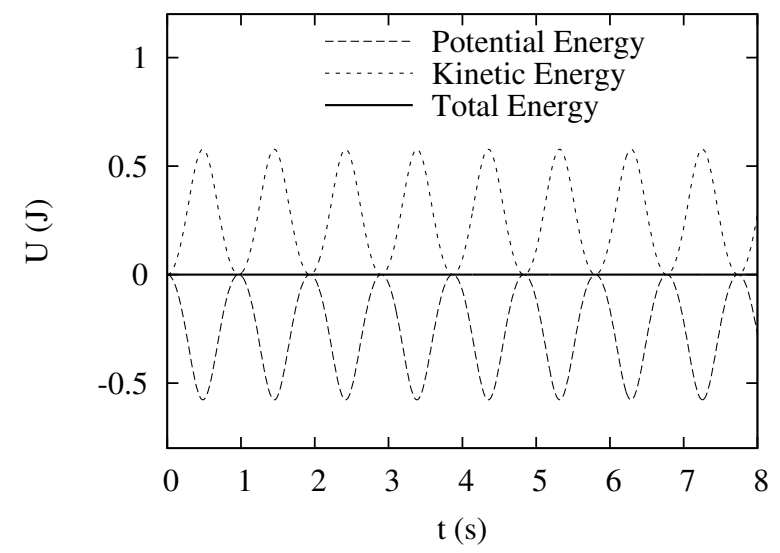

Figure 5: Energy of a falling rod with Young's modulus $E=$ $210 \mathrm{GPa}$

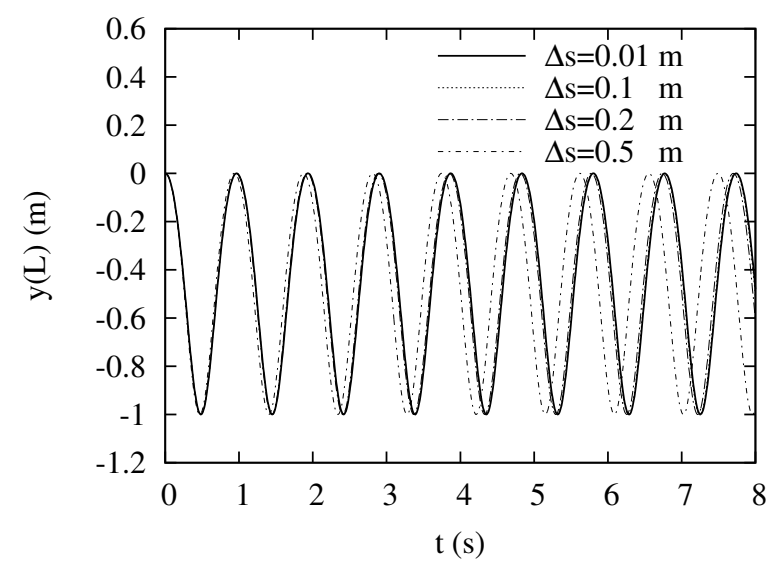

Figure 7: Convergence test by taking different spatial step sizes $\Delta s$ for a falling rod with Young's modulus $E=210 \mathrm{GPa}$.

Table 1: Stretch of the total length of the rod.

\begin{tabular}{ccccc}
\hline Young's modulus & $E=210 \mathrm{GPa}$ & $E=4 \mathrm{GPa}$ & $E=0.25 \mathrm{GPa}$ & $E=0.05 \mathrm{GPa}$ \\
\hline stretch & $1.0 \times 10^{-7}$ & $6.0 \times 10^{-7}$ & $1.0 \times 10^{-4}$ & $2.5 \times 10^{-3}$ \\
\hline
\end{tabular}




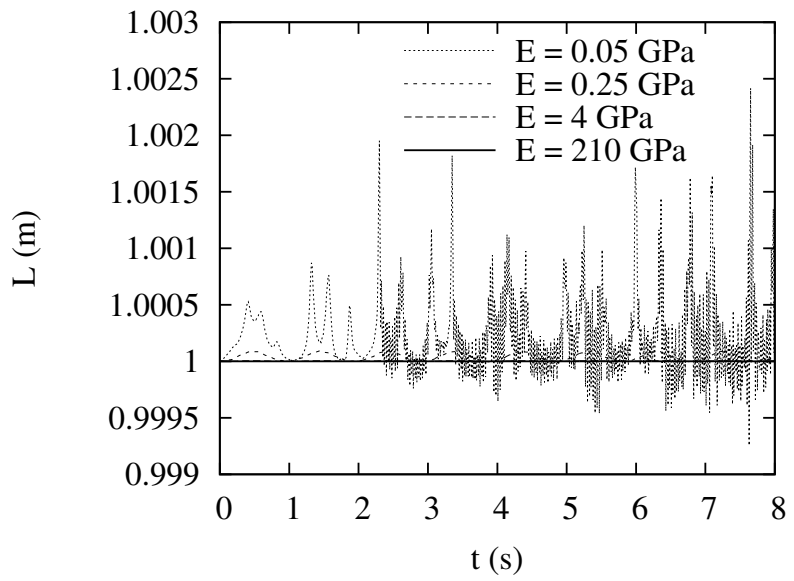

Figure 8: Total length of the stretched rod.
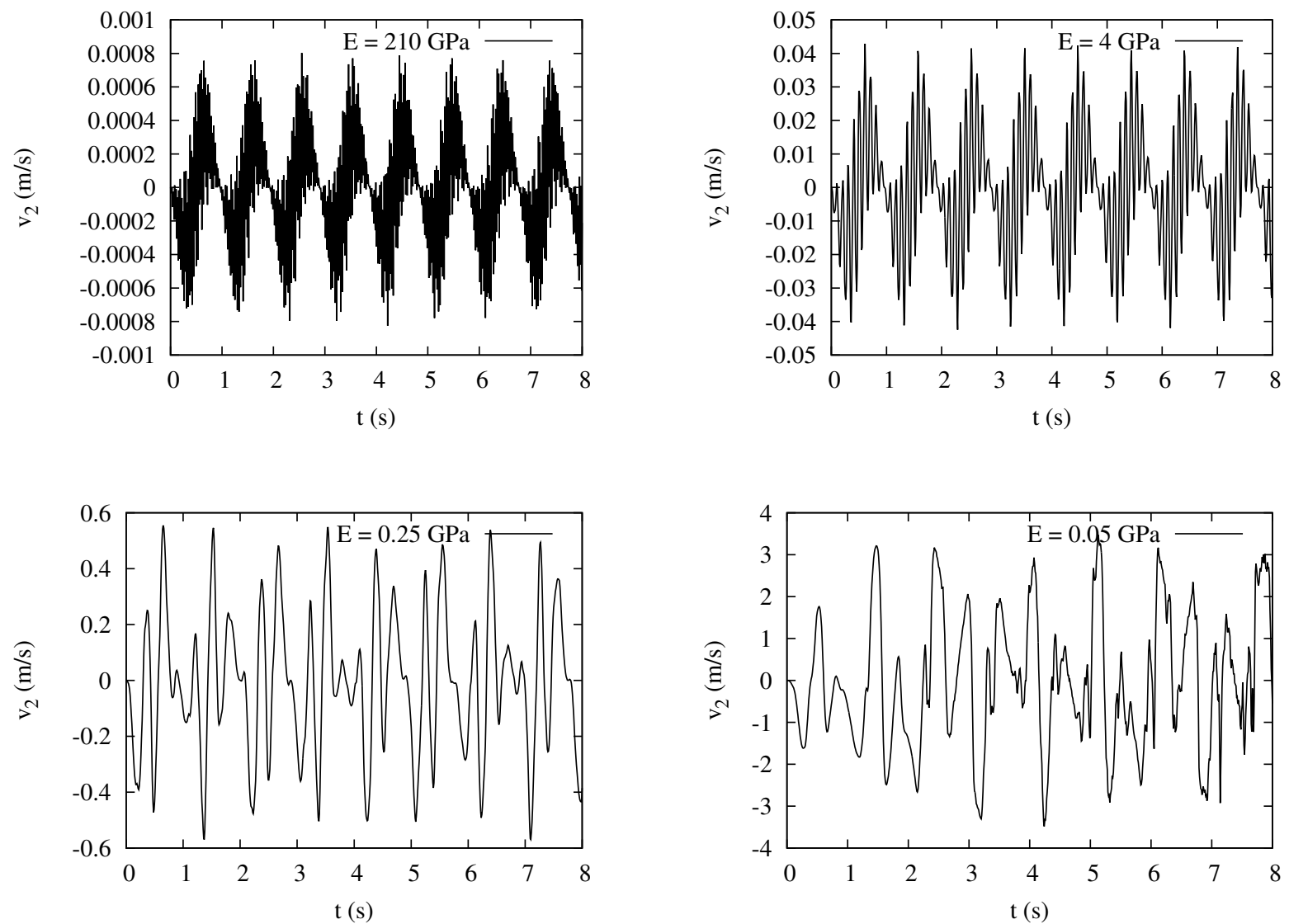

Figure 9: Plots of the $\mathbf{d}_{2}$ component of the end velocity, $u_{2}(L)$. 


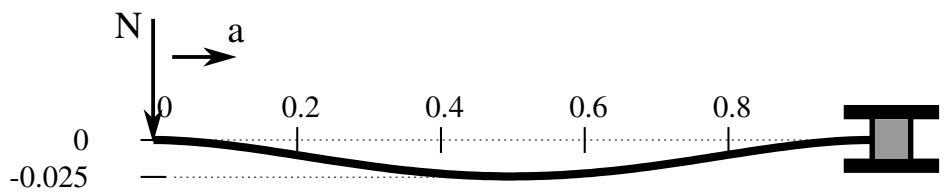

Figure 10: Initial configuration of the hanging cable $(N=20 \mathrm{~N})$.
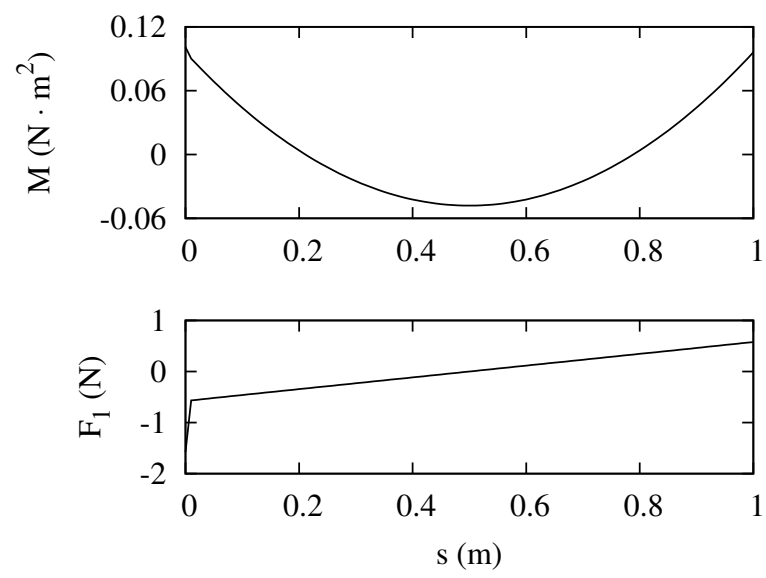

Figure 11: Internal moment and normal force of the initial rod under a point load $(N=2.0 \mathrm{~N})$.

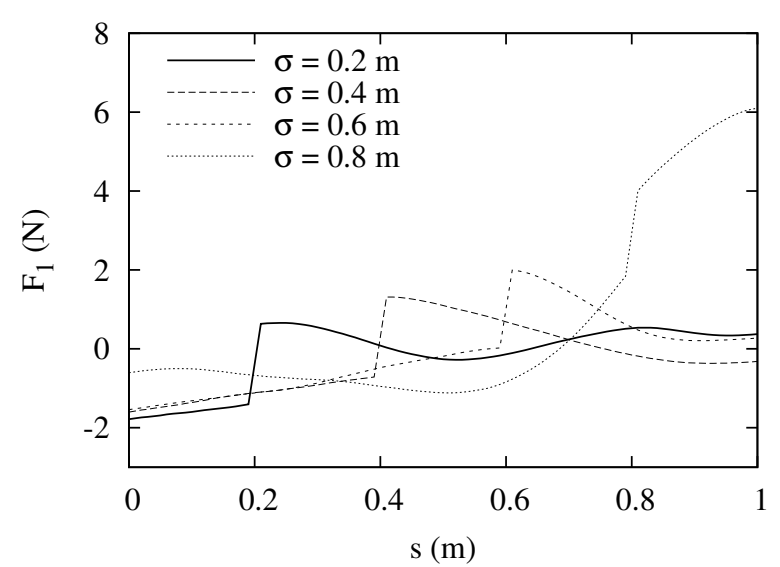

Figure 12: Internal normal force of the rod under a moving point load $(N=2.0 \mathrm{~N}, \alpha=1)$.

a point load $N$ at $s=0 \mathrm{~m}$ as the initial conditions for the dynamics problem (see Figs. 10 and 11). Putting the point load initially (at $t=0$ ) at the fixed support at $s=0$ mitigates ill numerical effects associated with the usual space-time corner singularities in time-dependent partial differential equations [34]. All runs for this example are carried out with $\Delta s=0.01$ and $\Delta t=\Delta s / a$.

At time $t=0 \mathrm{~s}$ the vertical load $N$ starts to travel from the left to the right end with constant speed $a$. This, for instance, models the motion of inspection robots along bridge cables or high-voltage power lines [21]. We consider two cases: a small load $(F=2.0 \mathrm{~N})$ and a large load $(F=20.0 \mathrm{~N})$. The point load causes a travelling discontinuity in the internal force in the rod as illustrated (for the small load) in Fig 12, where the shear force component $F_{1}$ is shown for various values of the time-like 'rod coordinate' $\sigma$, i.e., the instantaneous arclength position of the travelling load. Fig. 12 shows that our numerical method tracks and preserves the jump discontinuity of the moving load without difficulty (a jump discontinuity also occurs in the tension in the rod, $F_{2}$, while the bending moment, $M$, has a corner discontinuity). There is no sign of any oscillations indicative of the Gibbs phenomenon (and note that this is despite there being no damping in the system, not even numerical damping).

Figs. 13 and 14 compare the vertical midspan positions $x(L / 2)$ as a function of the rod coordinate $\sigma$ for the small- and large-load case. Curves are shown for various speeds $a$ given as a fraction $\alpha$ of the first critical speed $a_{1}$. For small vibrations this critical speed is the speed required to cross the cable in half a period of its first bending mode, i.e., $a_{1}=\omega_{1} L / \pi$, where for fixed-fixed boundary conditions the first natural frequency $\omega_{1}$ is given by $\omega_{1}=(4.7300 / L)^{2} \sqrt{E I / \rho A}$. We observe that in the case of a small load (Fig. 13) there is a resonance phenomenon in the sense that the maximum amplitude of vibrations occurs for speeds near the resonance speed $(\alpha=1)$. The rod effectively behaves as a stiff beam and the results agree with those for an Euler-Bernoulli beam found in [35]. In the case of a large load (Fig. 14) we observe a significant detuning effect: the rod behaves as a flexible cable with no resonance effect shown. The dimensionless parameter controlling 


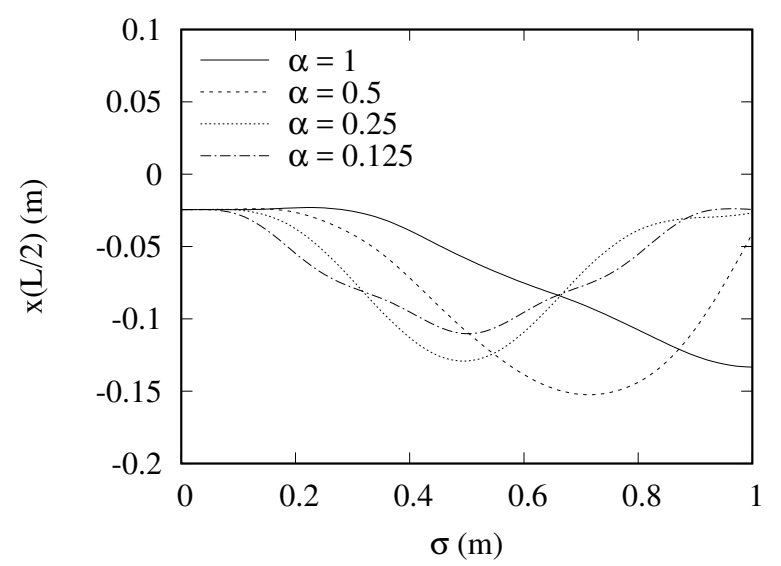

Figure 13: Midspan positions for various speeds $a$ given as a fraction $\alpha$ of the critical speed $(F=2.0 \mathrm{~N})$.

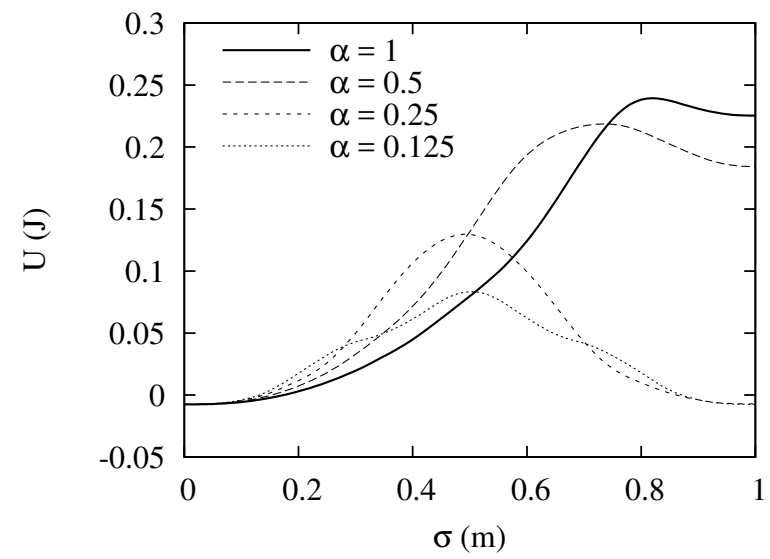

Figure 15: Rod total energy for various speeds $a$ given as a fraction $\alpha$ of the critical speed $(F=2.0 \mathrm{~N})$.

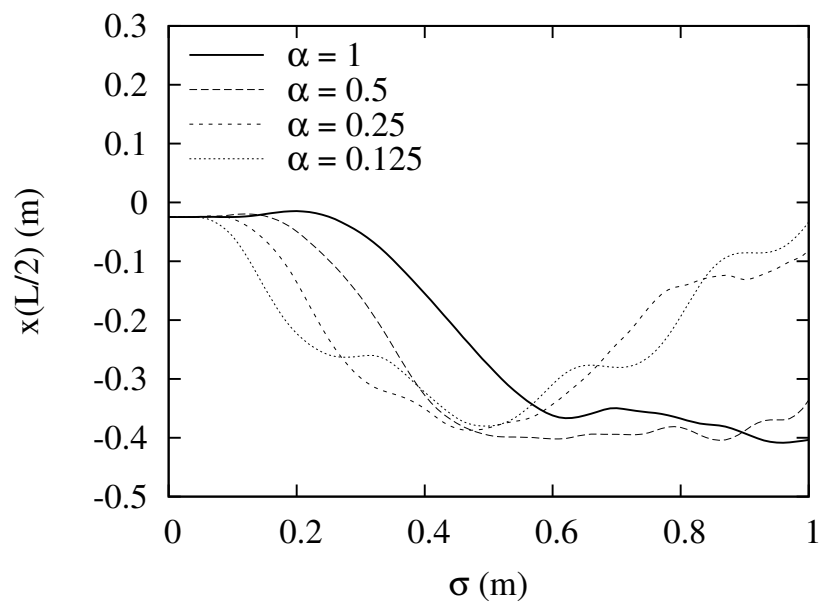

Figure 14: Midspan positions for various speeds $a$ given as a fraction $\alpha$ of the critical speed $(F=20.0 \mathrm{~N})$.

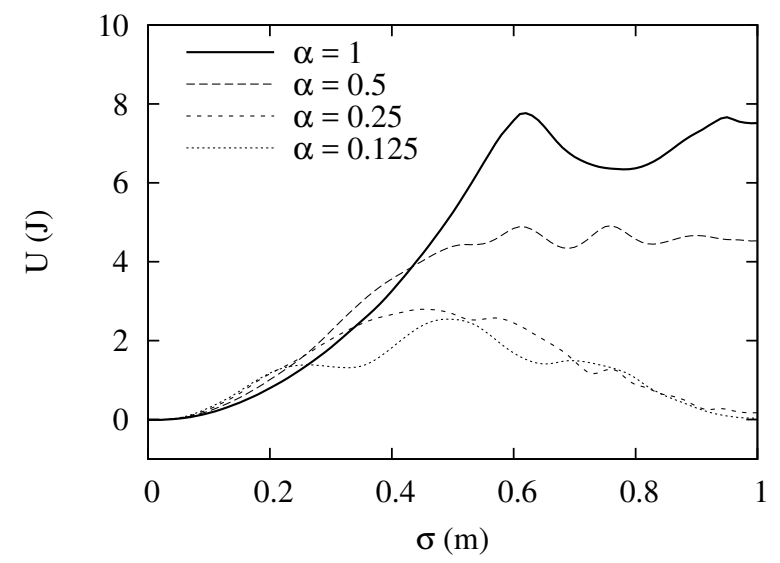

Figure 16: Rod total energy for various speeds $a$ given as a fraction $\alpha$ of the critical speed $(F=20.0 \mathrm{~N})$.

the effective stiffness/flexibility of the loaded cable is $E I / N L^{2}$.

Figs. 15 and 16 show the total energy $U$ of the rod as a function of $\sigma$ for the two load cases. The nonconservation of energy as the load travels along the rod nicely illustrates the Timoshenko paradox [36, 37], which can be stated as follows. A beam with horizontal end supports carrying a moving mass (or load), even though initially at rest, will be vibrating after the mass has left the beam, despite the fact that its weight will have done no work as the mass entered and left the beam at the same height. Where has the energy come from? The resolution of the paradox is well-known and is of course that the constant speed of the load, along the vibrating rod, would require some kind of control mechanism that would do work (against horizontal forces). The rod/moving-load system is therefore not a closed system and hence not conservative and Figs. 15 and 16 quantify the energy amplification (pumping) effect of the moving load on the rod. The effect in our present case of large-deflection rods is of course greater than in the traditional case of small-deflection beams. The energy initially increases monotonically in both load cases, but then goes through a maximum (when various couplings kick in). The energy remains highest when the load moves at resonance speed $(\alpha=1)$. After the load has left the $\operatorname{rod}($ at $\sigma=1$ ) the total energy remains constant, frozen at the value attained at $\sigma=1$. Figs. 17 and 18 give the rod shapes at various positions of the moving load. 


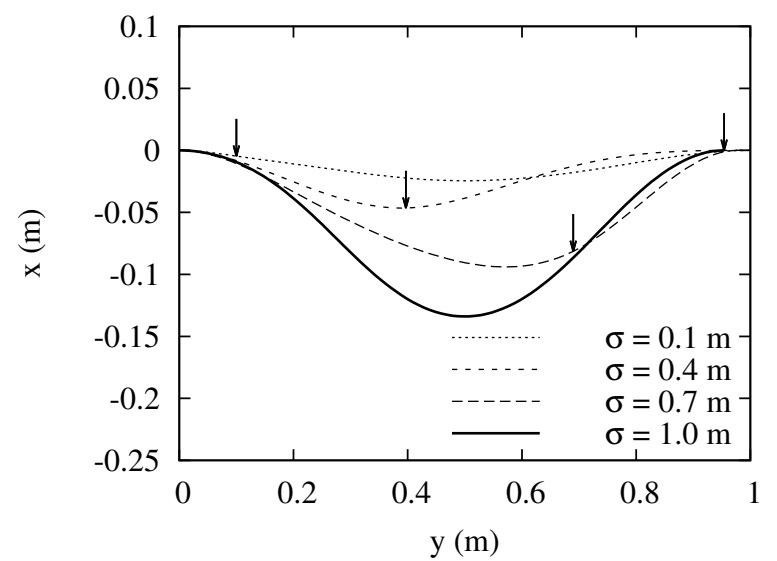

Figure 17: Rod shapes for various $\sigma(F=2.0 \mathrm{~N}, \alpha=1)$. Arrows indicate the position of the load.

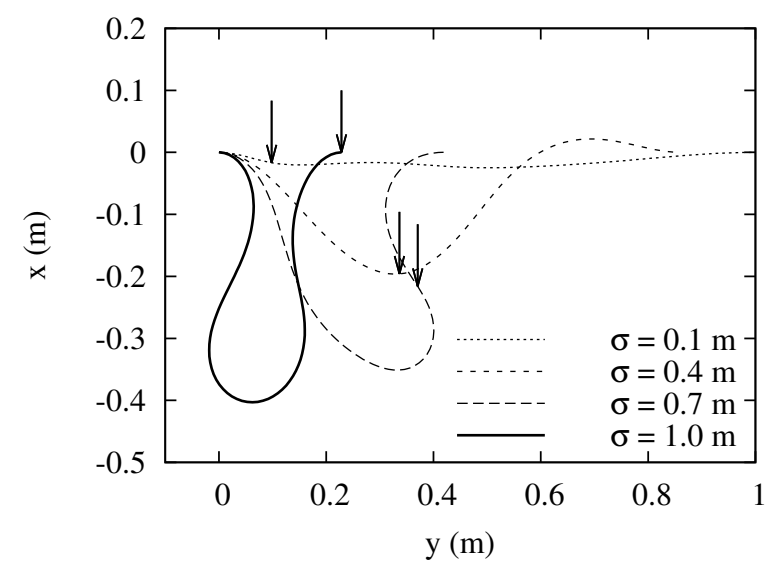

Figure 18: Rod shapes for various $\sigma(F=20.0 \mathrm{~N}, \alpha=1)$. Arrows indicate the position of the load.

\subsection{An arch under a moving load}

We consider an inextensible and unshearable arch with constant intrinsic curvature $\kappa_{0}=\pi / 2 \mathrm{~m}^{-1}$ (taking $E=0.25 \mathrm{GPa})$. With the given length $(L=1 \mathrm{~m})$ and arch radius $\left(R=1 / \kappa_{0}\right)$, the radian of the curved arch is $L / R$. We take the arch to be hinged at both ends. The boundary conditions of this problem are given by

$$
\begin{aligned}
s=0: & M=0, \quad y=0, \quad z=0, \\
s=L: & M=0, \quad y=0, \quad z=2 R \sin \frac{L}{R} .
\end{aligned}
$$

We again prepare the initial solution by solving the problem of a static arch under gravity and a point load at $s=0$ (setting $\mathbf{M}=\mathbf{0}$ and $\mathbf{M}_{c}=\mathbf{0}$ ). The true view of the static arch is shown in Fig. 19, while the bending moment and shear force (with end discontinuity) are plotted in Fig. 20. Fig. 21 shows the travelling jump discontinuity in the shear force at various values of $\sigma$.

Arches are well known to fail under sufficiently large point loads (see [38] for a recent study of in-plane collapse under a concentrated load applied at the centre of the arch). The maximum load an arch can sustain depends on the location where the load is applied. In Fig. 22 we present a bifurcation diagram, computed using the statics equations, showing the vertical position $x(\sigma)$ of the arch at the location $\sigma$ where the load $(N=2.5 \mathrm{~N})$ is applied as a function of $\sigma$. We observe a fold at $\sigma=\sigma_{c}=0.2863$. This means that under quasi-statically increasing $\sigma$ the arch fails at $\sigma=\sigma_{c}$, i.e., when the load has traversed $29 \%$ of the length of the arch. Doing the same calculation for different loads $N$ gives the failure curve in Fig. 23, where arch failure

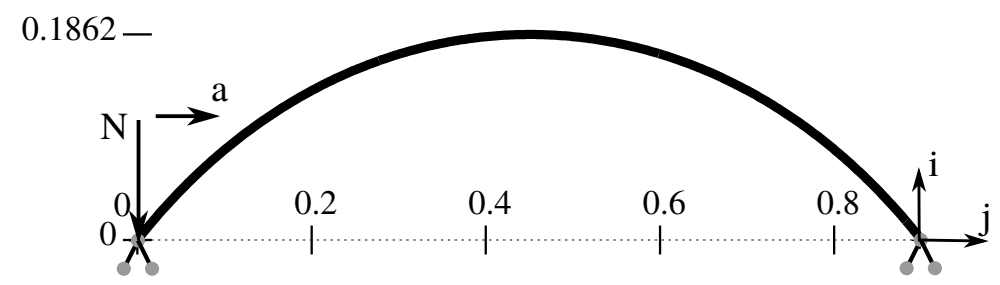

Figure 19: Initial configuration of an intrinsically-curved arch under the action of gravity and point load $N=2.5 \mathrm{~N}$ at $s=0 \mathrm{~m}$. 

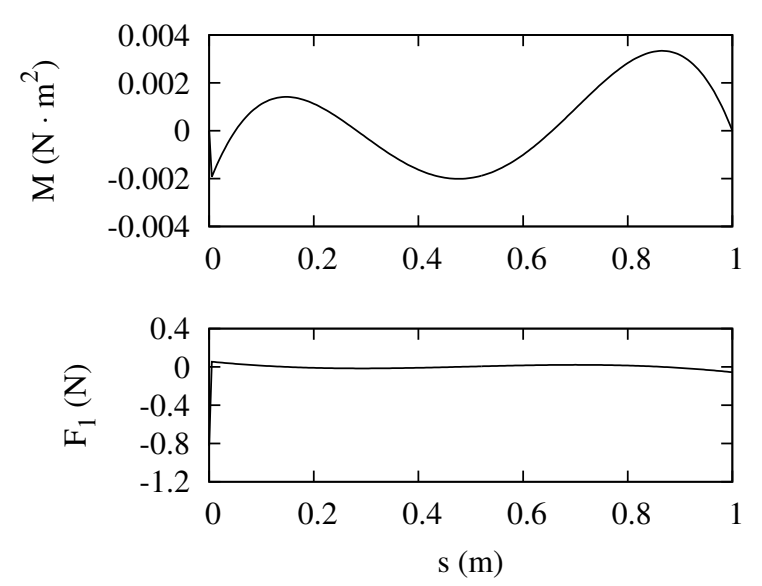

Figure 20: Bending moment $M$ and shear force $F_{1}$ of the static arch under a point load $(N=2.5 \mathrm{~N})$.

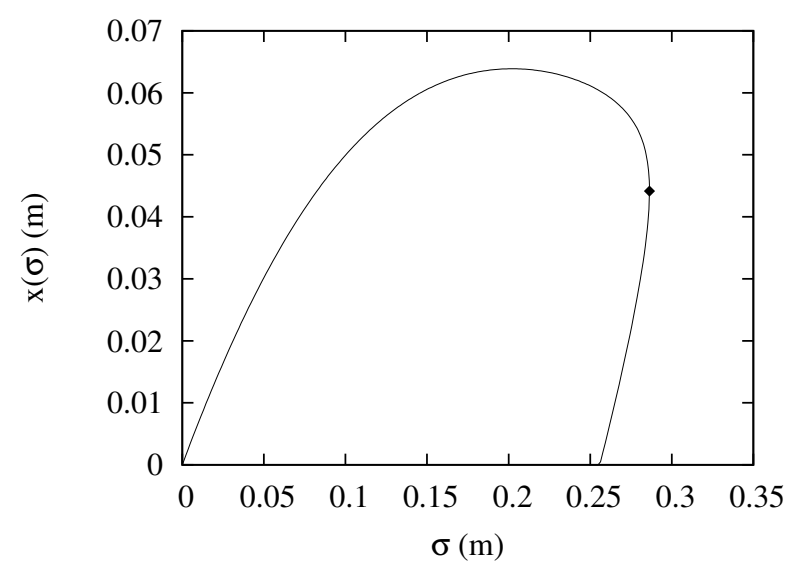

Figure 22: Bifurcation diagram for the hinged arch under quasistatic point load with the collapse point indicated $(N=2.5 \mathrm{~N})$.

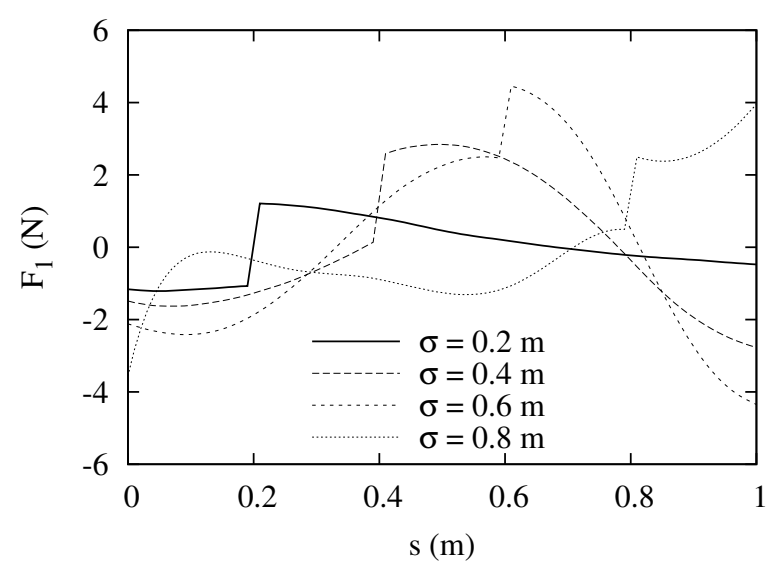

Figure 21: Internal normal force of the intrinsically curved arch under a moving point load ( $N=2.5 \mathrm{~N}, a=2.5 \mathrm{~m} / \mathrm{s})$.

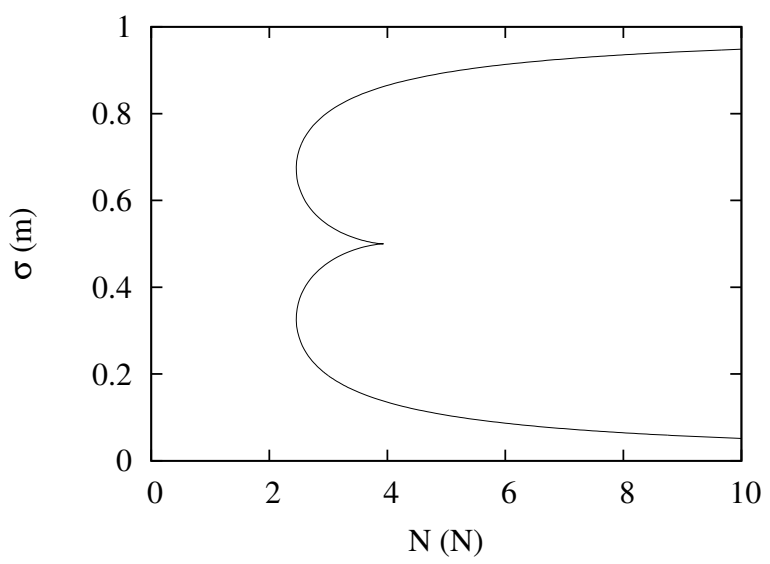

Figure 23: Failure diagram for a hinged arch under quasi-static point load. 


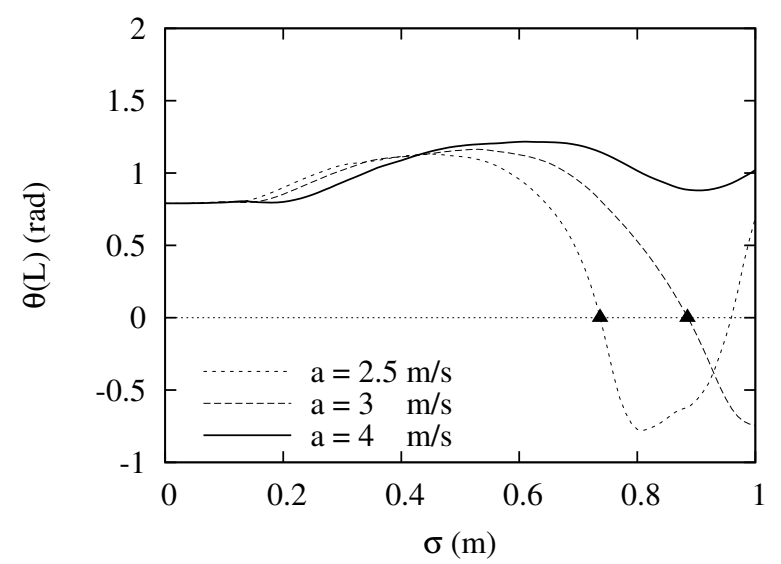

Figure 24: Plot of the end angle $\theta(L)$ of the $\operatorname{arch}(N=2.5 \mathrm{~N})$. The triangles indicate arch failure.

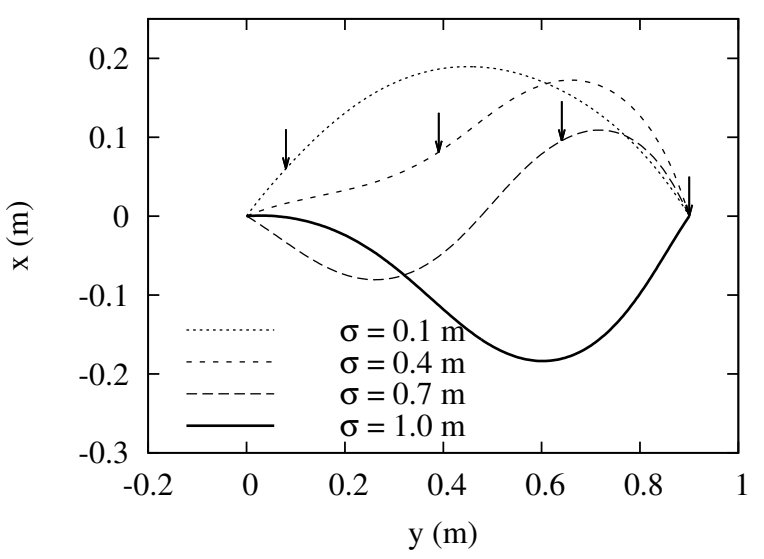

Figure 26: Shapes of the arch under a moving load $(N=2.5 \mathrm{~N}$, $a=3 \mathrm{~m} / \mathrm{s}$ ). Arrows indicate the position of the load.

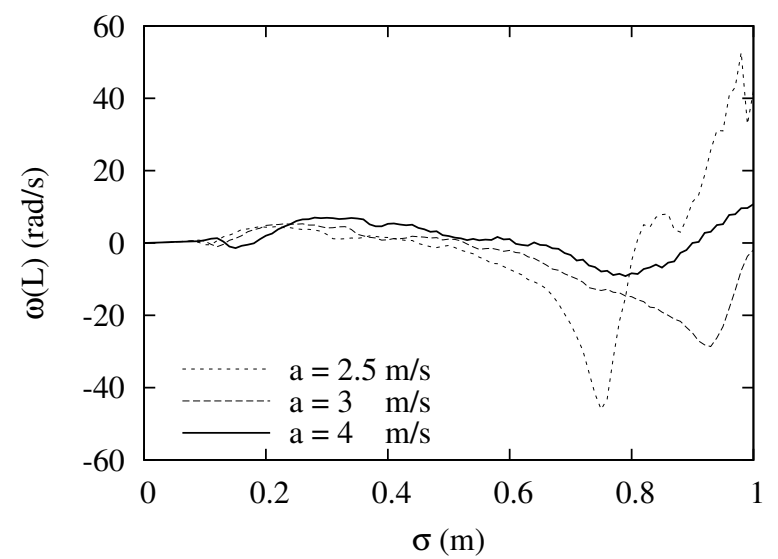

Figure 25: Plot of the end angular velocity $\omega(L)$ of the arch $(N=2.5 \mathrm{~N})$.

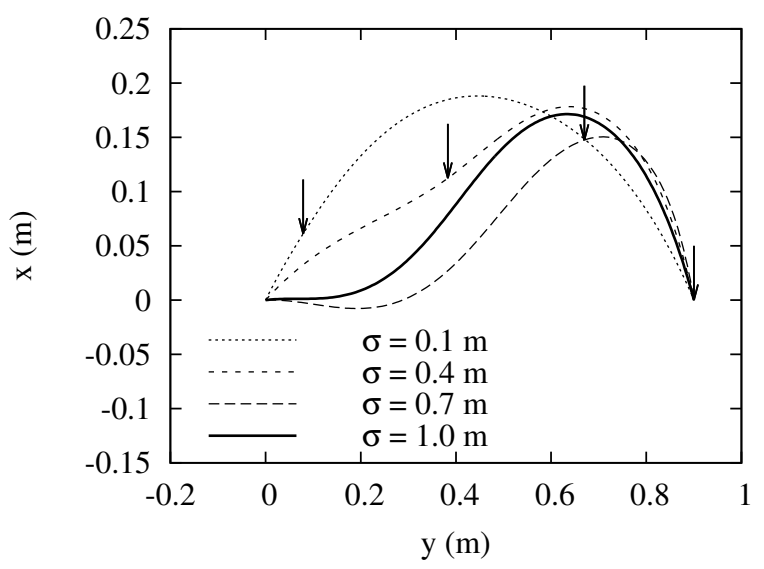

Figure 27: Shapes of the arch under a moving load $(N=2.5 \mathrm{~N}$, $a=4 \mathrm{~m} / \mathrm{s}$ ). Arrows indicate the position of the load.

occurs for parameters to the right of the curve. The figure reveals that the arch is weakest when the load is aplied at about a third along its length. Failure is here by in-plane collapse.

We now investigate the effect of the speed of the load on this arch failure. We let the same load $(N=2.5 \mathrm{~N})$ move from the left end with constant speed $a$. Results are shown in Figs 24-27. We take the end rotation angle $\theta(L)$ as the indicator of arch failure. When this angle first goes through zero from positive to negative we deem the arch to have collapsed (snapped through to an inverted shape). Fig. 26 and Fig. 27 present snapshots of true arch shapes when the point load is at different arclength positions $\sigma$. Fig. 24 clearly shows a delay effect on arch collapse due to the speed of the load by tracking the first point where $\theta(L)$ goes negative, indicated by the triangles. At speed $a=2.5 \mathrm{~m} / \mathrm{s}$ the load can move to position $\sigma=0.737 \mathrm{~m}$, at speed $a=3 \mathrm{~m} / \mathrm{s}$ it can move to position $\sigma=0.885 \mathrm{~m}$, while at speed $a=4 \mathrm{~m} / \mathrm{s}$ no collapse occurs at all. Angular velocity at the end, $\omega(L)$, is another indicator of arch failure. We notice that in Fig. $25 \omega(L)$ decreases rapidly when the arch fails, especially when the moving speed is slow, and varies more smoothly when the moving speed is increased.

In these runs we take $\Delta s=0.01 \mathrm{~m}$ and $\Delta t=\Delta s / a$ to sample the moving load at exact spatial nodes at each discretised time step.

\section{Conclusion}

We have formulated the problem of a slender structure undergoing arbitrarily large deformations under a moving load. The formulation is based on the geometrically exact Cosserat theory and the resulting partial 
differential equations are discretised by using the (second-order accurate) generalised- $\alpha$ method in both space and time. After testing the numerical implementation on the problem of a falling pendulum, we have applied our method to two typical slender structures, a cable and an arch. In both cases we find that the jump discontinuity as a result of a concentrated load is handled well; there is no sign of the Gibbs phenomenon typically plaguing formulations based on Galerkin approximation. Generalisation to multiple loads is straightforward.

The applications show interesting new phenonmena. We find that for a flexible cable large deformations induced by a moving load have a significant detuning effect on cable vibrations compared to the classical moving-load problem for a stiff beam. The cable problem also provides a compelling illustration of the Timoshenko paradox. For the arch problem we find a stabilising effect of the moving load. Buckling/collapse of a statically unstable arch is delayed by a moving load and suppressed altogether at sufficiently high speed.

The moving-load assumption does not take into account the inertial forces of the moving mass and the interactions between the moving mass and the continuous beam. The moving-load treatment is therefore insufficient when the inertial (Coriolis and centrifugal) effects of the moving mass are not negligible compared to the acceleration of the structure. Also, we have assumed prescribed motion of the load/mass (in fact, uniform speed along the rod in all our examples). This assumption will be unrealistic in certain applications with large deformations and a full theory will ultimately have to describe the two-way coupling between mass and structure. These issues will be addressed in ongoing work.

\section{Appendix - Jacobian}

The full set of nonlinear algebraic equations $\mathcal{H}$ in Eq. (34) is constructed from $\mathcal{F}_{j} \in \mathbb{R}^{6\left(N_{s}-1\right)}(j=$ $1, \cdots, N_{s}$ ) and $\mathcal{G}_{1} \in \mathbb{R}^{3}$. The full Jacobian takes the form

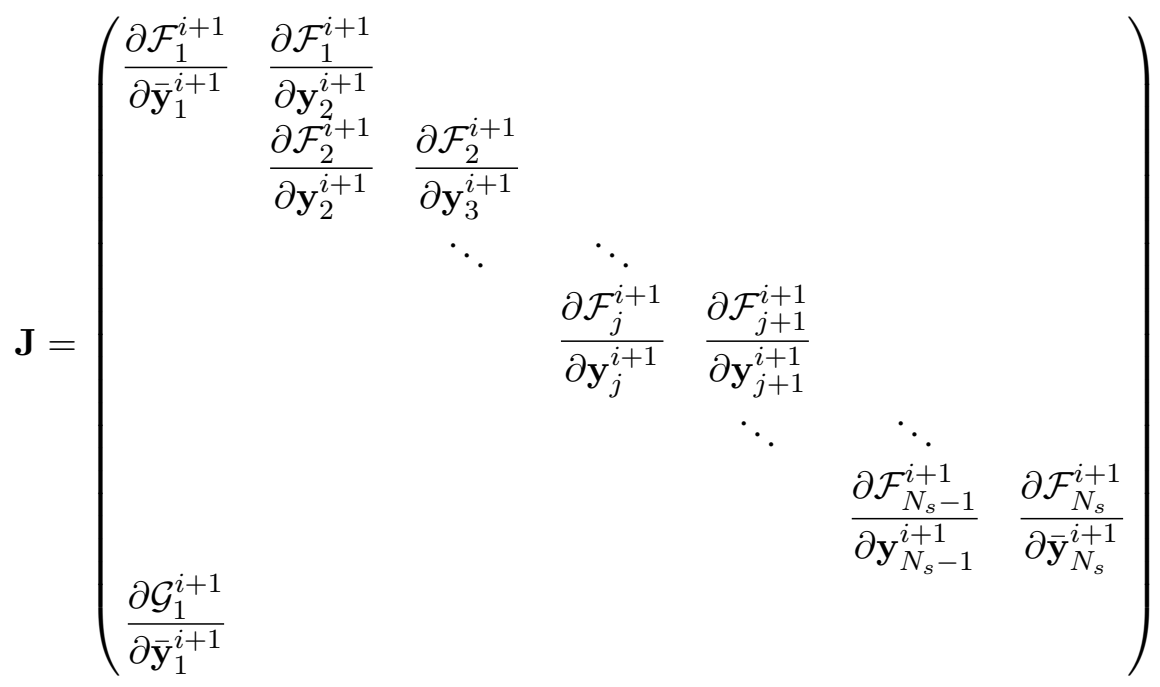

where

$$
\begin{aligned}
\frac{\partial \mathcal{F}_{j}^{i+1}}{\partial \mathbf{y}_{j+1}^{i+1}}= & p\left(1-\alpha_{s}\right) \mathbf{M}+q \mathbf{K}+h\left(1-\beta_{s}\right) \frac{\partial \boldsymbol{\Lambda}\left(\mathbf{y}_{j+1}^{i+1}\right)}{\partial \mathbf{y}_{j+1}^{i+1}} \\
\frac{\partial \mathcal{F}_{j}^{i+1}}{\partial \mathbf{y}_{j}^{i+1}}= & p \alpha_{s} \mathbf{M}-q \mathbf{K}+h \beta_{s} \frac{\partial \boldsymbol{\Lambda}\left(\mathbf{y}_{j}^{i+1}\right)}{\partial \mathbf{y}_{j}^{i+1}}, \\
& \frac{\partial \mathcal{G}_{j}^{i+1}}{\partial \mathbf{y}_{j}^{i+1}}=p \mathbf{M}_{c}+h \frac{\partial \boldsymbol{\Lambda}_{c}\left(\mathbf{y}_{j}^{i+1}\right)}{\partial \mathbf{y}_{j}^{i+1}},
\end{aligned}
$$

and

$$
p=\frac{1-\alpha_{t}}{\gamma_{t} \Delta t}, \quad q=\frac{\left(1-\beta_{t}\right)\left(1-\beta_{s}\right)}{\gamma_{s} \Delta s}, \quad h=1-\beta_{t} .
$$

Note that $\overline{\mathbf{y}}$ denotes variables at boundaries. 


\section{References}

[1] P.K.C. Wang, J. Wei, Vibrations in a moving flexible robot arm, Journal of Sound and Vibration 116 (1987) 149-160.

[18] A. Sofi, G. Muscolino, Dynamic analysis of suspended cables carrying moving oscillators, International Journal of Solids and Structures 44 (2007) 6725-6743.

[19] L. Wang, G. Rega, Modelling and transient planar dynamics of suspended cables with moving mass, International Journal of Solids and Structures 47 (2010) 2733-2744. 
[36] S. Timoshenko, Vibration Problems in Engineering, Van Nostrand, New York, 1937.

[37] L. Maunder, Paradoxes, Mechanism and Machine Theory 89 (2014) 38-42.

[38] J. Zhu, M.M. Attard, D.C. Kellermann, In-plane nonlinear buckling of circular arches including shear deformations, Archive of Applied Mechanics 84 (2014) 1841-1860. 